\title{
Safety Enhancement Framework for Robotic Minimally Invasive Surgery
}

\author{
V. Penza ${ }^{1,2}$, E. De Momi ${ }^{2}$, N. Enayati ${ }^{2}$, T. Chupin ${ }^{2}$, J. Ortiz ${ }^{1}$, L. S. Mattos ${ }^{1}$ \\ ${ }^{1}$ Department of Advanced Robotics, Istituto Italiano di Tecnologia, Genoa, Italy \\ ${ }^{2}$ Department of Electronics, Information and Bioengineering, Politecnico di Milano, Italy \\ veronica.penza@polimi.it
}

\section{INTRODUCTION}

In abdominal surgery, intra-operative bleeding is one of the major complications that affect the outcome of minimally invasive surgical procedures, even when performed with a robotic system. One of the causes is attributed to accidental damages to vessels, and one of the possible risk factors falls on surgeon's skills [1].

Computer-assisted technologies coupled with surgical robotic systems can enhance surgeons' capabilities by providing additional information regarding the surgical gestures. The intra-operative identification of vessels to be preserved has been explored using pre-operative information registered on the patient and visualized by means of Augmented Reality (AR) [2]. However, this approach has to deal with dynamic changes of the anatomy between the data acquisition phase and the surgical procedure. In order to update the preoperative/intra-operative registration at run-time, imagebased stereo reconstruction and tracking algorithms can be exploited to describe the tissue deformations [3]. Although such computer vision algorithms have achieved considerable performance, what is still missing is their integration into a surgical robotic system, providing (i) high accuracy, (ii) long-term robustness in difficult circumstances (field of view occlusion, presence of blood or smoke, sudden camera movements), (iii) adaptation to various changes of the environment or of the object itself and (iv) real-time processing.

This paper presents an Enhanced Vision System to Improve Safety in Robotic Surgery (EnViSoRS) by warning the surgeon in case an instrument is approaching a delicate area to be protected. This area, also defined Safety Area (SA), can be intra-operatively identified by the surgeon on the image. A tracking algorithm is used to robustly track the $\mathrm{SA}$, and consequently, the tissue surface is reconstructed. The $3 \mathrm{D}$ information is used to identify a Safety Volume (SV) fitted around the reconstructed tissue surface. AR is used to visualize at run-time the SV projected on the image and to display the tissue-instrument distance. The system has been integrated into the da Vinci Research Kit (dVRK, provided by WPI and Johns Hopkins University) to validate its usability under realistic conditions.

\section{MATERIALS AND METHODS}

An overview of EnViSoRS integrated in the dVRK is shown in Fig. 1. The framework consists of five main steps, which are described in the following paragraphs.

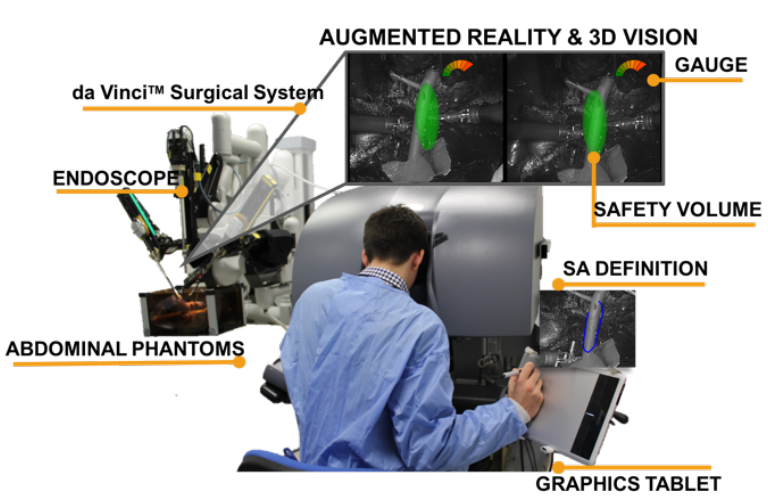

Fig. 1 EnViSoRS system: Enhanced Vision System to improve Safety in Robotic Surgery integrated into dVRK system (WPI and Johns Hopkins University). From the console the surgeon can (i) select the SA using a stylus and a graphics tablet, (ii) see the SV overlaid on the images, and (iii) see a graphical gauge warning about the tissue-instruments distance.

\section{Step 1: Read Images}

In this state, image left and right are captured, the distortion is corrected and the images are rectified in order to facilitate the search for stereo correspondence of the $3 \mathrm{D}$ reconstruction method. Specular highlights are also identified as bright regions with low saturation and high intensity to prevent errors in feature tracking and 3D reconstruction computation.

\section{Step 2: Safety Area Definition}

The $\mathrm{SA}$ is a $2 \mathrm{D}$ polygon that can be defined by the surgeon on the image at any moment of the surgery. A digital drawing tablet with stylus (WACOM Bamboo Pen and Touch Tablet) was placed on one side of the da Vinci ${ }^{\mathrm{TM}}$ master console, allowing the surgeons to perform this operation while they are seated, viewing the images from the stereo-endoscope.

\section{Step 3: Tissue Tracking}

The Long-Term Safety Area Tracking (LT-SAT) algorithm [4] is used to robustly track the SA when (i) the endoscopic camera is moved by the surgeon to navigate the operating field, (ii) the tissues are moving or deforming, or (iii) the field of view is occluded by instruments or smoke. The algorithm combines an optical flow technique with a tracking-by-detection approach to improve the robustness against failures. A Bayesian inference-based approach is used to detect tracking failures and a Model Update Strategy (MUpS) is exploited to improve the SA redetection after a failure. 


\section{Step 4: Tissue 3D Reconstruction}

A dense soft tissue stereo-reconstruction algorithm [5] is used to retrieve the 3D surface of the tissue contained in the SA. To reduce the computational time, the tracked SA is used as a prior for the coarse identification of the area of the image to reconstruct. A pixel-based foregroundbackground segmentation is used to discard the $3 \mathrm{D}$ points laying on the background. The algorithm is based on a block matching approach, exploiting a non-parametric Census transform to make the stereo-matching robust to illumination variations. The density of the reconstructed surface is improved by using Simple Linear Iterative Clustering (SLIC) super pixel during the disparity refinement. With respect to the previous implementation, a novel strategy is proposed to smooth the point cloud. The disparity image is considered as a 2D Laplace equation problem. The disparity values on the super pixel contours are considered as the Dirichlet boundary conditions and the remaining as the initial conditions. The Gauss-Seidel method with red and black ordering is used to solve the equations.

\section{Step 5: Safety Augmentation}

The aim of this state is (i) to visualize the projection of the SV fitting the reconstructed surface of the SA, and (ii) to warn the surgeon when the robotic instruments are approaching the tissue surface. The SV is computed as an ellipsoid fitted on the point cloud and its $2 \mathrm{D}$ projection is displayed on the image by means of AR. In order to know the distance between the end-effector of the instruments and the reconstructed surface, spatial neighbor search based on octree structure is used. If the distance is inside the range $\Delta_{\text {dist }}$, the surgeon is visually warned through a gauge located in the top-right corner of the image, as shown in Fig. 2.

\section{RESULTS}

To validate the robustness and usability of EnViSoRS, experimental trials were performed involving 14 subjects and 3 surgeons specialized in robotic surgery in urology. The task they performed consisted in tele-operating the da Vinci arms to move a piece of tissue from one side of a liver phantom to another and then moving it back, paying attention to keep a certain distance from a vessel positioned in the middle of the operating field, as shown in Fig. 2. Each subject performed 2 sessions, respectively with and without EnViSoRS; for each session the task was repeated 2 times. The operating field used was a previously developed phantom of human abdomen [6]. The robustness of the system was evaluated verifying the overlap between the SV projected on the image as AR and the SA visible in the image. The precision and recall curves, computed varying the overlap ratio threshold, gave an Area Under the Curve (AUC) of 0.898 and 0.932, respectively. The usability of EnViSoRS was evaluated asking the subjects to fill the System Usability Scale (SUS) questionnaire, which allows the evaluation of surgical technology in terms of general usability. Results showed a higher global score using EnViSoRS w.r.t. using the standard da Vinci system without AR feedback

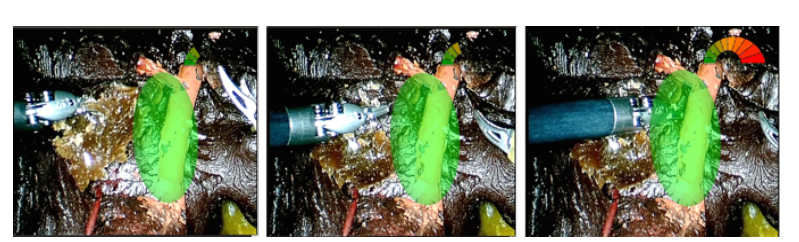

Fig. 2 Example of AR visualization. From left to right, three different situations showing (i) the instruments performing the surgery in the safe green range, (i) the right instrument approaching the delicate area, (iii) the left instrument almost touching the vessel surface and the gauge completely red.

(79.21 vs 74.74 in the range $0-100)$. The performance in terms of computational time showed an average frame rate between $2.5 \mathrm{fps}$ to $4 \mathrm{fps}$.

\section{DISCUSSION}

This paper demonstrates the high robustness, usability and interactive frame rate of EnViSoRS, a computer vision framework aimed at improving the safety during robotic minimally invasive surgery. The added value of this work is the integration of EnViSoRS with dVRK system, showing the application into a state-of-the art robotic surgical system, thus shortening the distance to a future real clinical application of the technology. Future work will aim at improving the system under different aspects: (i) defining active constraints by using haptic feedback to guide the surgical gesture, (ii) exploring different modalities to define the SA and represent the $\mathrm{AR}$ information, and (iii) speeding up the computation time, exploiting CPU-GPU processing.

This project has received funding from the European Union's Horizon 2020 research and innovation programme under grant agreement No. H2020-ICT-2016- 732515.

\section{REFERENCES}

[1] I. Opitz, W. Gantert, U. Giger, T. Kocher, L. Krähenbühl, et al. Bleeding remains a major complication during laparoscopic surgery: analysis of the salts database. Langenbeck's Archives of Surgery. 2005; 390:128-133.

[2] S. Onda, T. Okamoto, M. Kanehira, F. Suzuki, R. Ito, S. Fujioka, et al. Identification of inferior pancreaticoduodenal artery during pancreaticoduodenectomy using augmented reality-based navigation system. Journal of hepato-biliarypancreatic sciences. 2014; 21:281-287.

[3] D. Stoyanov, D. Surgical vision. Annals of biomedical engineering. 2012; 40:332-345.

[4] V. Penza, X. Du, D. Stoyanov, A. Forgione, L. Mattos, and E. De Momi. Long term safety area tracking (LT-SAT) with online failure detection and recovery for robotic minimally invasive surgery. Medical Image Analysis (under review).

[5] V. Penza, J. Ortiz, L. Mattos, A. Forgione E. and De Momi. Dense soft tissue $3 \mathrm{D}$ reconstruction refined with superpixel segmentation for robotic abdominal surgery. International journal of computer assisted radiology and surgery. 2015:11: 197-206.

[6] A. Ciullo, V. Penza, L. Mattos, and E. De Momi. Development of a surgical stereo endoscopic image dataset for validating $3 \mathrm{~d}$ stereo reconstruction algorithms. 6th Joint Workshop on New Technologies for Computer/Robot Assisted Surgery. 2016. 